\title{
The role of parieto-temporal connectivity in pure neglect dyslexia
}

\author{
Radek Ptak ${ }^{1,2,3}$, Marie Di Pietro ${ }^{1}$ and Jean-Michel Pignat ${ }^{4}$
}

${ }^{1}$ Division of Neurorehabilitation, Department of Clinical Neurosciences, University Hospitals Geneva, Geneva, Switzerland

${ }^{2}$ Laboratory of Cognitive Neurorehabilitation, Faculty of Medicine, University of Geneva, Switzerland ${ }^{3}$ Faculty of Psychology and Educational Sciences, University of Geneva, Geneva, Switzerland

${ }^{4}$ Unit of Acute Neurorehabilitation, Department of Clinical Neurosciences, University Hospital Lausanne, 1011 Lausanne, Switzerland

\section{Corresponding author:}

Radek Ptak, PhD

Division of Neurorehabilitation

Geneva University Hospitals

26, Av. de Beau-Séjour, 1211 Geneva 14, Switzerland

Tel.: +41-22-372 3524

radek.ptak@hcuge.ch 


\section{ABSTRACT}

The initial stages of reading are characterized by parallel and effortless access to letters constituting a word. Neglect dyslexia is an acquired reading disorder characterized by omission or substitution of the initial or the final letters of words. Rarely, the disorder appears in a 'pure' form that is, without other signs of spatial neglect. Neglect dyslexia is linked to damage involving the inferior parietal lobe and regions of the temporal lobe, but the precise anatomical basis of the pure form of the disorder is unknown. Here, we show that pure neglect dyslexia is associated with decreased structural connectivity between the inferior parietal and lateral temporal lobe. We examined patient DM, who following bilateral occipitoparietal damage presented left neglect dyslexia together with right visual field loss, but no signs of spatial neglect. DM's reading errors were affected by word length and were much more frequent for pseudowords than for existing words. Most errors were omissions or substitutions of the first or second letter, and the spatial distribution of errors was similar for stimuli presented left or right of fixation. The brain lesions of DM comprised the inferior and superior parietal lobule as well as the cuneus and precuneus of the left hemisphere, and the angular gyrus and lateral occipital cortex of the right hemisphere. Diffusion tensor imaging revealed bilateral decrease of fibre tracts connecting the inferior parietal lobule with the superior and middle temporal cortex. These findings suggest that parieto-temporal connections play a significant role for the deployment of attention within words during reading.

Keywords: Visual attention; reading; neglect dyslexia; spatial neglect; parietal lobe; temporal lobe; disconnection.

\section{INTRODUCTION}

Perceiving and acting toward objects requires computing representations that relate the spatial coordinates of the object to the viewer (egocentric coding) or to other objects in the environment (allocentric coding). In an egocentric reference system the horizontal and vertical coordinates of the 
stimulus are defined with respect to the viewpoint of the observer while in an allocentric system they are independent of a subjective viewpoint. The study of spatially lateralized deficits of visual attention such as extinction or neglect has strongly contributed to the understanding of how the human brain processes spatial relationships between objects and the viewer or the environment (Halligan et al., 2003; Hillis, 2006). The study of neglect dyslexia, a reading disorder affecting the letters of a word that are located opposite to a unilateral brain damage, has provided particularly abundant findings concerning spatial reference systems in the brain (Ellis et al., 1987; Vallar et al., 2010). Neglect dyslexia is characterized by omissions or substitutions of the initial or final letters of words, leading to neologisms or the replacement of a word by another. Given that words are almost always presented in a canonical view and reading always proceeds in the same direction one may expect words to be coded exclusively in an egocentric reference system. However, based on David Marr's (1982) model of the coding of visual objects and on observations of patients with distinct patterns of reading disorders Hillis and Caramazza (1995) proposed that words (as a special case of visual 'objects') are coded in distinct spatial reference frames. At the most basic level of representation - the viewercentred frame - the position of the word is related to a vertical axis defined by the viewer's body, head or retina. Given that the position of the word is specified with respect to the viewer, this representation is crucial for the programming of eye movements during reading. The simplest way to test whether neglect dyslexia depends on a viewer-centred representation is to show words at different horizontal positions. A purely viewer-centred deficit would be expected if the neglected proportion of letters were greater for words placed more contralesionally respective to the egocentric viewpoint. At the next level, the cognitive representation is defined by the intrinsic vertical axis of the word, and is therefore independent of the word's position respective to the viewer. The representation is stimulus-centred, which means that reading errors will not depend on the horizontal position of the word: a comparable proportion of letters will be neglected for words placed in left or right space (Ellis et al., 1987; Hillis and Caramazza, 1991). Finally, at the most abstract level of representation - the word-centred frame - the intrinsic coordinates of the word are not only independent relative to the viewer, but also its orientation in space. A deficit affecting this representation will become manifest in neglect errors that are independent of whether words are 
presented horizontally or rotated (Caramazza and Hillis, 1990; Miceli and Capasso, 2001). This model has the advantage that it allows understanding partly the heterogeneity of error patterns presented by different patients with neglect dyslexia.

In one of the first papers on neglect dyslexia Ellis et al. (1987) concluded that the disorder has no specificity, but is a simple consequence of other neglect symptoms. Indeed, while the presence of neglect is not a systematic predictor of neglect dyslexia (Behrmann et al., 2002), most patients with this disorder also exhibit other signs of neglect (Vallar et al., 2010) or may show disturbed oculomotor behaviour that is not confined to reading (Primativo et al., 2013; Primativo et al., 2015). Among the classic paper-and-pencil tests used to assess neglect line bisection appears to be the only significant predictor of neglect dyslexia (Ptak et al., 2012; Reinhart et al., 2013). Nevertheless, several case studies of 'pure' neglect dyslexia (i.e., dyslexia without other neglect symptoms; Arduino et al., 2005; Haywood and Coltheart, 2001; Riddoch et al., 1990) or of dyslexia that is dissociated from other signs of neglect (e.g., left dyslexia with right neglect in cancellation tasks; Costello and Warrington, 1987; Cubelli et al., 1991; Humphreys and Riddoch, 1995) have been reported. However, all patients classified as 'pure' neglect dyslexics had at least partial visual field loss that may have influenced the reading disorder, in particular if combined with subclinical symptoms of neglect. Thus, the degree of interdependence between neglect dyslexia, visual field loss and other neglect symptoms is not entirely clear.

Another relevant question regarding neglect dyslexia concerns its anatomical correlates. Of particular interest are anatomical dissociations between neglect dyslexia (as special form of 'stimulus-centred' deficit) and viewer-centred deficits, which would support the independence of egocentric and allocentric representations in the brain. Unfortunately, anatomical correlates have not been the focus of previous reports of pure neglect dyslexia. However, two group studies examined lesion location in right-hemisphere damaged neglect patients with signs of dyslexia, compared to neglect patients without the reading disorder. Lee et al. (2009) reported that neglect dyslexia was selectively associated with damage to the right fusiform and lingual gyrus, though the lesions in their patients also encroached upon more dorsal regions including the inferior parietal cortex. We performed a similar study on 19 patients with neglect dyslexia and found that the disorder was best predicted by a 
combination of damage to the posterior parietal lobe (including the angular gyrus and the intraparietal sulcus) and middle and inferior temporal gyri (Ptak et al., 2012). This finding suggests that stimuluscentred reading deficits may be a consequence of combined damage to the dorsal and the ventral stream of visual information processing (Ungerleider and Mishkin, 1982; Ungerleider and Pasternak, 2003).

Here, we studied a patient with pure neglect dyslexia following bilateral occipito-parietal lesions. Despite the presence of right homonymous hemianopia reading errors concerned the left side of the stimulus. A detailed structural imaging and tractography study revealed bilateral disconnection between damaged inferior parietal cortex and preserved middle/superior temporal cortex. These findings indicate that stimulus-centred deficits in reading result from parieto-temporal disconnection.

\section{MATERIALS AND METHODS}

\subsection{Case report}

DM, a 45 years old, right-handed (lateralization index: 100; Oldfield, 1971) coachbuilder suffered bilateral cerebral infarctions due to disseminated intravascular coagulation following manifestation of acute myeloid leukaemia. MRI showed a large left parietal lesion as well as a smaller right inferior parietal and superior occipital lesion. The patient had complete right hemianopia and initially showed visuo-perceptual deficits characterized by optic ataxia, impossibility to detect or localize visual targets when several distracters were present (simultanagnosia) as well as slight difficulty disengaging gaze from a fixated object (ocular apraxia). Reading was very difficult and frustrating for the patient. The signs of a Bálint syndrome regressed within the first weeks and the patient regained his independence in most activities of daily life. However, he continued to exhibit difficulty with reading, often transforming the beginning of a word (e.g., he would read the word 'distraction' as 'contraction'). The present study was performed two years following onset of the symptoms. At this time the patient was completely independent in daily life and showed excellent compensation of his right visual field loss. Approval for this study was obtained from the ethical committee of the University Hospitals Geneva, and DM gave written informed consent. 
A neuropsychological examination including detailed assessment of DM's visual and visual-spatial impairments was performed 1.5 years following injury. The patient had an average verbal IQ (94), but low performance IQ (68) at the WAIS (Wechsler, 2008) due to impaired performance on picture completion, digit-symbol and block design subtests. He had normal spoken language including naming (100\% correct on the Boston naming test), praxis and verbal and nonverbal recognition memory (Warrington, 1984). Humphrey Perimetry revealed a right homonymous hemianopia with macular sparing of $\sim 5$ degrees in the upper quadrant (Figure 1). The patient had normal colour perception as tested with the Ishihara test and a short form of the Farnsworth test, and normal object (including identification of silhouettes, Table 1) and face perception (Benton faces test). Table 1 shows that DM had moderate to severe impairments of visual-spatial functions while he scored comparatively better in object identification. He had no neglect on cancellation tasks (always starting on the left), or complex figure drawing, and line bisection performance was only slightly biased (compatible with slight over-compensation towards the side of hemianopia), but remained within the range of healthy controls (Ronchi et al., 2012). On a variant of the Posner task (Zimmermann and Fimm, 2010) the patient showed no signs of a disengagement deficit (Table 1) which is strongly associated with left neglect (Losier and Klein, 2001; Morrow and Ratcliff, 1988). Together, these findings indicate that though DM had non-lateralized visual-spatial impairments, he had no sign of left neglect in classic paper-and-pencil tests or of a deficit of attentional disengagement.

\section{Insert Table 1 about here}

Insert Figure 1 about here

\subsection{Stimuli}


Two sets of words and matched non-words were used for the experiments. Set 1 was constructed in order to test linguistic factors such as word length (in terms of the number of letters), frequency and lexical status. It comprised 180 words of 3-10 letters, 1-4 syllables and 0-25 orthographic neighbours, and a mean frequency of 78.5 per thousand according to the Lexique database of written French (http://www.lexique.org; New et al., 2004). For each word a pseudoword was created by replacing randomly 1-2 letters while preserving the consonant/vowel status and thus length and orthotactic status. A sub-set of these words (42 words, 42 pseudowords) was used to test vertical reading. Set 2 was composed of 906 -letter nouns half of which were selected in an attempt to maximize the number of neglect dyslexia errors and to study the effects of stimulus position on reading performance. The Lexique database was searched for 6-letter nouns with frequent endings (i.e., at least 3 nouns found in the database shared the same ending) or non-frequent endings (i.e., only one word was found with this ending). This search returned a series of words for each frequent ending (e.g. 35 words for the ending 'ANT', such as mutant, enfant, savant) and one word for each nonfrequent ending (e.g. the word azimut for the ending 'MUT'). We then searched in the frequentendings list words whose frequency matched the frequency of one of the rare-endings words. In this way we created two lists of 45 words where the lexical frequency of the frequent and non-frequent endings items was matched one to one (1.12 per thousand for the frequent-endings words; 1.13 per thousand for the rare-endings words), but the number of orthographic neighbours sharing only the word ending with the target differed ( 15.11 for the frequent-endings words; 0.2 for the rare-endings words). We additionally created for each of the 90 words a pseudoword by transposing the first three letters while respecting the orthotactic status (e.g. 'socmos' for cosmos). All stimuli were written in 20-point lower-case Arial font and subtended a horizontal size of 2.3 - 5.8 degrees, a 6-letter word subtending 3.5 degrees (see Figure 1).

\subsection{Procedure}

Words were presented on a 20" flat screen with a resolution of $1024 \times 768$. The patient sat at an approximate distance of $57 \mathrm{~cm}$ from the screen. In all experiments a black fixation cross $(0.5$ degrees) was presented between trials on the screen. When the experimenter pressed a mouse 
button the fixation cross was immediately replaced by a word. The patient was asked to read aloud the word and his answer was registered through a head-set for later off-line analysis.

In a first step we tested the effects of linguistic variables such as lexicality, word length and frequency on the distribution of DM's reading errors using stimulus set 1. All 180 words and 180 pseudowords were presented centrally, once with unlimited presentation time and once at $400 \mathrm{~ms}$. In a second step we used stimulus set 2 to examine the spatial distribution of reading errors as a function of presentation time and position of the word on the screen. The stimuli were presented unmasked for $400 \mathrm{~ms}, 50 \mathrm{~ms}$ or $13 \mathrm{~ms}$ (which was the fastest presentation time possible with our screen). Finally, DM was again tested with set 2 words presented at one of three positions: centrally or with the word centre located at 6 degrees to the left or right of fixation. The position of the stimulus was selected randomly and was therefore unpredictable for the patient. The aim of this experiment was to test to what extent neglect dyslexia errors of DM depended on an egocentric frame of reference. Our prediction was that if errors depended exclusively on a stimulus-centred representation the patient should show the same frequency and pattern of neglect dyslexia errors irrespective of the position of the stimulus on the screen. In contrast, a dependence of neglect dyslexia errors on horizontal position would indicate that the reading disorder of DM depended on a viewer-centred reference frame (Hillis and Caramazza, 1995).

\subsection{Structural and diffusion tensor imaging}

A high-resolution T1-weighted MRI 3D gradient-echo with magnetization preparation (MPRAGE) sequence (TR: 2500; TE: 3; slice resolution: $1.1 \mathrm{~mm}$ ) was acquired on a 3-T scanner (Siemens Medical Solutions, Erlangen, Germany). DM was scanned 17 months following brain injury. A lesion mask was delineated directly on the MRI scan using a graphics tablet and MRIcron (Rorden et al., 2007), and the brain of the patient was segmented and normalized to standard space with help of the clinical toolbox (www.mricro.com/clinical-toolbox; Rorden et al., 2012), which uses SPM8's (www.fil.ion.ucl.ac.uk/spm) unified normalization/segmentation function.

For the tractography study 18 healthy males age-matched to the patient (mean age difference, $-4.2 \pm$ 11.7 years) were recruited as controls. In addition, connectivity was evaluated in 5 patients with left 
hemisphere and 5 patients with right hemisphere lesions. Patients were older than DM (age, $61.8 \pm$ 8.1 years) and none of them had impaired reading though three patients with right-hemisphere damage showed moderate to severe neglect in a cancellation task. Neuroimaging data were acquired in the context of a previous study (Guggisberg et al., 2015).

DTI acquisition consisted of a spin-echo planar imaging (EPI) sequence using monopolar diffusion gradients in 30 directions (b-value $=1000 \mathrm{~s} / \mathrm{mm} 2$ ). Axial slices were acquired with slice thickness of 2 $\mathrm{mm}$ and in-plane resolution of $1.8 \mathrm{~mm}^{2}$ (TR: $8200 \mathrm{~ms}$; TE: $82 \mathrm{~ms}$ ). The number of fibres between pairs of regions of interest (ROIs) was compared between the patient and the control group. All number of fibres values were embedded in normalized non-oriented connectivity matrices, which were generated from the diffusion MRI data using Connectome Mapper (http://www.cmtk.org/mapper/; Daducci et al., 2012). Full processing encompassed several stages: (1) segmentation of the grey matter into 83 parcels according to the Desikan-Killiany anatomical atlas (Desikan et al., 2006); (2) registration of the segmented data to the diffusion space; (3) processing of whole-brain tractography by means of a probabilistic algorithm to decrease sensitivity to noise and cumulative propagation errors, and (4) connectivity matrix estimation by combining whole-brain tractography expressed in number of fibres with the cortico-subcortical parcellation results. The last step provides an 83 x 83 cell matrix depicting all pairwise ROI connections. Disruption of connectivity was evaluated between the following ROIs: the cuneus and precuneus, the supramarginal, inferior parietal, latero-occipital, middle frontal, superior frontal, middle temporal and superior temporal region. For the statistical analysis, the number of fibres of the patient's brain was first subtracted from the value of each control subject, resulting in a set of 18 differences for each pair of ROIs. As all comparisons were normally distributed, we performed t-tests to examine whether connectivity differed significantly between the patient and the control groups. The Bonferroni-Holm method (Holm, 1979) was used to correct p-values for multiple comparisons.

Insert Figure 2 about here 


\section{RESULTS}

\subsection{Reading}

DM made 30 errors (8.3\%) during unlimited presentation of word set 1 compared to 104 errors (28.9\%) when presentation time was limited to 400 ms. Given the small number of errors with unlimited presentation we only analysed the 400 ms condition. There was a strong effect of lexicality, as only 20 errors concerned words (19.2\%), the remaining being made on pseudowords (80.8\%). Following Ellis et al. (1987) neglect dyslexia errors were categorized as substitutions, omissions or additions of letters to the left of a clearly identifiable neglect point within the word. This criterion has been used in numerous previous studies, but has the disadvantage that it does not consider errors that concern the centre or right, but not the left side of the word. We therefore additionally verified for each letter of the word whether it was subject to a substitution or omission error (Martelli et al., 2011). In order to simplify comparison across words of different length we divided each stimulus into a left, central and right part.

Out of the 104 errors, 9 were complete word misses, and 76 were left neglect dyslexia errors according to the Ellis et al. (1987) criteria: 45 pure substitution, 12 omission, 11 mixed substitution/omission, 4 mixed substitution/addition, and 4 pure addition errors. The remaining 19 errors concerned mixtures of substitutions or omissions of left, central or right letters. A letter-based analysis showed that out of all 92 letter substitutions across the word, $71.7 \%$ concerned the first two letters, $13 \%$ the last two letters and $15.2 \%$ letters in between. Word length affected DM's errors, as the average length of correctly identified items (6.25 letters) was significantly shorter than the length of misspelled items $\left(7.17\right.$ letters; $\left.t_{358}=3.46, p<.001\right)$. In contrast, there was no effect of word frequency: frequency of misspelled words (74.9) was comparable to the frequency of correctly identified words $\left(78.9 ; \mathrm{t}_{178}=0.12\right)$.

Using word set 2 (6-letter words with selected word-endings) we first tested the effect of presentation time. At $400 \mathrm{~ms} 79 \%$ of all errors were neglect dyslexia errors, while at shorter presentation times this rate dropped significantly ( $46 \%$ at $50 \mathrm{~ms} ; 16 \%$ at $13 \mathrm{~ms}$ ), while the number of non-responses 
correspondingly increased (400 ms: $21 \%$; $50 \mathrm{~ms}: 54 \%$; $13 \mathrm{~ms}: 84 \%$ ). Presentation time thus overall decreased the number of neglect dyslexia errors, but did not shift within-word position of errors. We next tested reading with words presented at different positions on the screen $(-6,0$ or +6 degrees) for $400 \mathrm{~ms}$, which allowed testing the following predictions: If neglect dyslexia of DM depended on the position on screen (i.e., was viewer-centred), we would expect an increase of the rate of dyslexia errors when words are presented further to the left. If the rate of reading errors remained constant, the patient's dyslexia would be classified as stimulus-centred. On the other hand, given DM's right-sided hemianopia the patient might produce reading errors on the final letters of words when the stimulus was presented in his right visual field. In order to examine the within-word distribution of reading errors we counted the number of letter substitutions, omissions and additions of the two beginning letters, the two central or the two final letters of each word.

Out of 1080 stimuli DM made 284 (26.3\%) left neglect dyslexia errors (that is, errors that concerned only the left or left and central letters), 201 (18.6\%) non-responses and 36 (3.3\%) errors that could not be classified. Lexical status was a strong predictor of errors, as $223(78.5 \%)$ of all errors were made on pseudowords, compared to $61(21.5 \%)$ errors on words $\left(X^{2}=125.4, p<.0001\right)$. Most neglect dyslexia errors were substitutions, followed by omissions and mixed errors, and this distribution was unaffected by lexical status (Figure 2A). A letter-based analysis showed that out of all 393 letter substitutions occurring at any position across the word, $60.8 \%$ concerned left letters, $36.6 \%$ central letters and only $2.5 \%$ right letters. Figure $2 \mathrm{~B}$ shows the distribution of neglect dyslexia errors as a function of the position of the word on screen. DM's rate of errors on the left and left/centre words (which are both classified as neglect dyslexia errors) was comparable for the three positions $\left(X^{2}=2.13, \mathrm{~ns}\right)$. As Figure $2 \mathrm{~B}$ shows the rate of errors made on the final letters (right and right/centre errors) was very low irrespective of word position on the screen, and was therefore not analysed.

Overall, the findings indicate that DM's neglect dyslexia was independent of a viewer-centred reference frame, suggesting that his deficit was either stimulus-centred or word-centred. In order to distinguish between both possibilities we tested whether reading of vertically presented words/pseudowords $(n=84)$ would result in a similar pattern of neglect dyslexia errors as horizontal 
presentation. DM found the task difficult and made 34 reading errors (40.5\%). His errors were difficult to analyse due to many substitutions and omissions, but mostly concerned the central and final letters of each item. DM identified the first letter correctly in $33(97 \%)$, the second letter in $30(88.2 \%)$, the second-last letter in $9(26.5 \%)$ and the last letter in $10(29.4 \%)$ instances. This pattern suggests that in vertical reading he proceeded sequentially (from the beginning to the end of the word), which is distinct from his pattern when reading horizontally presented words. Finally, when asked to spell 32 words and 16 pseudowords, DM made two autocorrected errors on words and two on pseudowords, only one of these errors concerning the beginning of the word (he spelled pied - foot as 'bied'). These findings indicate that DM's neglect dyslexia was not word-centred and was therefore best classified as stimulus-centred.

\section{Insert Figure $\mathbf{3}$ about here}

\subsection{Neuroimaging}

Figure 3 shows the reconstruction of DM's brain lesions. The patient suffered extensive posterior left hemispheric damage, affecting parts of the superior and inferior parietal lobule, the cuneus and the precuneus. Laterally, the lesion extended to the temporal-parietal junction and the posterior part of the superior temporal sulcus. In the right hemisphere damage was much smaller and affected the inferior part of the angular gyrus, as well as parts of the superior and lateral occipital cortex.

Whole-brain parcellation of the DTI data did not reveal loss of any of the studied ROls, indicating that they were at least partly preserved in the patient. Figure 4 shows the results of the connectivity analyses. In the left hemisphere the supramarginal gyrus was significantly less connected to the middle temporal (compared to healthy controls: $p<.01$; brain-damaged controls: $p<.01$ ) and the superior temporal gyrus $(p<.0001$ and $p<.05$, respectively). Similarly, the patient showed a loss of connectivity between the inferior parietal region and the middle temporal (compared to healthy controls: $p<.05$ and brain-damaged controls, $p<.0001$, respectively) and superior temporal gyrus (both control groups, $\mathrm{p}<.05$ ). In the right hemisphere a significant loss of connectivity was measured between the supramarginal gyrus and the middle temporal gyrus (healthy controls, $p<.0001$ and 
brain-damaged controls, $\mathrm{p}<.05)$ as well as between the inferior parietal region and the middle temporal gyrus (healthy controls, $p<.05$ ad brain-damaged controls, $p<.005$ ). In addition, only in the right hemisphere connections between the inferior parietal and the superior frontal region were also significantly disrupted as compared to healthy controls $(p<.005)$ and brain-injured controls $(p<$ $.0001)$.

\section{Insert Figure 4 about here}

\section{DISCUSSION}

The present patient showed left neglect dyslexia in the absence of any other sign of neglect. In none of the classic clinical tasks (such as cancellation, line bisection, or complex figure drawing) or in a spatial cueing task did DM show any signs of left neglect. This case supports some of the rare reports showing that neglect dyslexia may be present in the absence of other signs of spatial neglect (Arduino et al., 2005; Haywood and Coltheart, 2001; Riddoch et al., 1990) and adds new information concerning lesion anatomy and connectivity changes that may underlie this disorder.

DM's neglect dyslexia was characterized by several factors. First, reading time and lexicality were the two most important variables predicting the occurrence of neglect dyslexia reading errors. DM made few $(<10 \%)$ reading errors with unlimited presentation, against approximately one third of errors when presentation time was limited to $400 \mathrm{~ms}$. This is a duration at which healthy adults comfortably identify words of different lengths (Fisher, 1975). He also made many more errors when reading pseudowords ( $80 \%)$ than when reading words ( 20\%) and showed a significant effect of word length. Another characteristic of DM's reading concerns the frame of reference in which reading errors occurred. Presenting words to the left or right of fixation did not affect the frequency and position of reading errors. This finding has been described in several patients before (Haywood and Coltheart, 2001; Hillis and Caramazza, 1995; Katz and Sevush, 1989; Subbiah and Caramazza, 2000) and contrasts with other studies reporting significant effects of the retinal position of words on neglect dyslexia errors (Hillis et al., 1998; Riddoch et al., 1990). The effects of vertical presentation on DM's reading are slightly more complex to interpret, because the task was very difficult for DM. If 
his dyslexia were word-centred, we would expect him to make reading errors on the initial letters even for rotated words (Caramazza and Hillis, 1990; Miceli and Capasso, 2001). This was clearly not the case, as in vertical reading DM proceeded sequentially from top to bottom and made only few errors on word beginnings. Together, these findings indicate that DM's reading deficit was neither viewer-centred nor word-centred. The patient thus exhibited a pure form of stimulus-centred neglect dyslexia in the absence of spatial neglect.

All characteristics of DM's dyslexia described above have previously been observed in other neglect dyslexia patients. We emphasize this point because if DM's dyslexia were in some way special our anatomical findings would possibly reflect a particular case and may therefore apply only to a subgroup of neglect dyslexia patients. Our patient presented neglect dyslexia following bilateral posterior cerebral damage. A previous review of single case reports of neglect dyslexia revealed that in all 28 patients with acquired right-hemispheric lesions the deficit affected the beginning that is, the contralesional side of words (Vallar et al., 2010). In contrast, the pattern was more complicated for patients with left-sided damage. Only four out of 12 published cases had contralesional (right-sided) dyslexia, while the remaining eight patients made ipsilesional errors. In addition, five patients with neglect dyslexia following bilateral damage were published previously, and four of these patients made reading errors on the beginning of words. Interestingly, just as the present patient all lefthemisphere damaged patients with an ipsilesional reading disorder had a contralesional visual field defect. Based on these findings two anatomical interpretations of DM's reading disorder are possible. First, DM's neglect dyslexia may be a consequence of his right hemisphere damage to parts of the angular gyrus and the lateral occipital cortex. This interpretation is partly in line with our previous anatomical study showing that the brain regions differentiating right-hemisphere damaged neglect patients with dyslexia from patients without dyslexia are the inferior parietal cortex and the middle temporal gyrus (Ptak et al., 2012). On the other hand, the meta-analysis of Vallar, et al. (2010) and several group studies have shown that neglect dyslexia without other signs of neglect after righthemisphere damage is rare (Arduino et al., 2006; Làdavas et al., 1997; Lee et al., 2009; Stenneken et al., 2008), which questions our interpretation. 
Alternatively, DM's neglect dyslexia was due to his extensive left hemisphere damage, and was therefore ipsilateral to the lesion. This interpretation is plausible given that in most published cases of neglect dyslexia following left hemisphere damage (8 of 12 , i.e. $67 \%$ ) reading errors concerned ipsilateral letters (Binder et al., 1992; Cubelli et al., 1991; Katz and Sevush, 1989; Patterson and Wilson, 1990). This prevalence of ipsilesional deficits distinguishes left hemisphere from right hemisphere neglect dyslexia, as in the latter the deficit always appears to be contralesional (Vallar et al., 2010). A second important difference is that while all left-hemisphere patients with left dyslexia had no or right-sided scotoma or neglect, all except one right-hemisphere patient identified in the review of Vallar, et al. (2010) had spatial impairments affecting the same side as dyslexia (i.e., left). In our patient the side of visual field impairment and of neglect dyslexia dissociated, which is comparable to left-hemisphere damaged patients. Finally, the present case is reminiscent of the patient described by Arduino et al. (2005), who following bilateral temporo-occipital damage presented a stimulus-centred left neglect dyslexia. This patient showed right upper quadrantanopia with no signs of neglect other than in reading and cerebral hypoperfusion that was more important in the left than the right hemisphere, suggesting that his left-hemispheric lesion may have caused his reading impairment. Thus, though an anatomical interpretation should be accepted with caution these arguments favour the conclusion that DM's neglect dyslexia is a consequence of his left hemisphere lesion.

How do these findings relate to previous results regarding the anatomy of neglect dyslexia? Two anatomical studies of neglect dyslexia have produced somewhat contrasting results. Lee et al. (2009) compared 30 patients with left neglect and neglect dyslexia to neglect patients without dyslexia. They reported that damage to the right lingual and fusiform gyrus was a strong predictor of neglect dyslexia. However, closer inspection of their data shows that damage associated with neglect dyslexia also affected the lateral occipital cortex, the cuneus and parietal white matter. In a similar study involving 19 neglect dyslexia patients we identified the angular gyrus, the intraparietal sulcus and the middle temporal gyrus as the main predictors of the disorder (Ptak et al., 2012). These findings suggested that the inferior parietal and the lateral temporal cortex were involved in neglect dyslexia. The present case report supports these data by showing that pure neglect dyslexia is 
associated with a disconnection between the inferior parietal and lateral temporal cortex. Our patient showed weaker connections in the left and right hemisphere than healthy controls between the inferior parietal lobule and the superior and middle temporal gyrus. These findings suggest that damage to the inferior parietal lobe and the lateral temporal cortex or the disconnection between both regions is necessary to produce neglect dyslexia. Note that this conclusion holds even if our interpretation that DM's dyslexia is due to left hemisphere damage turned out to be wrong, as the patient also showed significant reduction of parieto-temporal fibre counts in the right hemisphere.

The inferior parietal lobe has been implicated in the shifting and disengagement of spatial attention, both on the basis of neuropsychological (Friedrich et al., 1998; Golay et al., 2008; Molenberghs et al., 2008; Ptak and Fellrath, 2013) and functional imaging findings (Indovina and Macaluso, 2007; Kincade et al., 2005; Serences et al., 2005; Shulman et al., 2009). These studies support the importance of the inferior parietal lobe for detection of unexpected, behaviourally relevant stimuli, and for attentional selection under conditions of competition in an egocentric reference frame. In contrast, studies examining which brain regions are important for the deployment of attention within objects (i.e., allocentric coding) support the importance of parts of the temporal lobe, such as the parahippocampal gyrus (Grimsen et al., 2008), the superior temporal sulcus and middle temporal gyrus (Chechlacz et al., 2010), or the posterior inferior temporal region (Medina et al., 2009). Interestingly, the lateral and inferior temporal cortex exhibits category-specific activity when subjects view pictures of tools or animals (Chao et al., 1999), suggesting that representations elaborated here rely on the coding of internal characteristics of objects. Such within-object coding differentiates ventral (lateral occipital, inferior and lateral temporal) cortex from between-object coding relying on inferior and superior parietal cortex (Humphreys, 1998). Object coding may additionally be affected by visual crowding, which is experienced when objects are composed of multiple local elements and may therefore contribute to occurrence of neglect dyslexia (Martelli et al., 2011).

In addition to processes involved in the computation of spatial reference frames, impaired withinobject deployment of attention due to the lack of access of inferior parietal regions to representations of internal object structure elaborated in the temporal lobe may contribute to stimulus-centred deficits 
such as neglect dyslexia. Our findings suggest that the coordination between dorsal and ventral visual streams plays a significant role for the peripheral stages of reading.

\section{Acknowledgement}

Study supported by the Swiss National Science Foundation (grant 320030-152689). We thank A. Guggisberg for granting access to neuroimaging data of the control groups. 


\section{REFERENCES}

Arduino, L.S., Daini, R., Silveri, M.C., 2005. A stimulus-centered reading disorder for words and numbers: Is it neglect dyslexia? Neurocase. 11, 405-415.

Arduino, L.S., Vallar, G., Burani, C., 2006. Left neglect dyslexia and the effect of stimulus duration. Neuropsychologia. 44, 662-665.

Behrmann, M., Black, S.E., McKeeff, T.J., Barton, J.J.S., 2002. Oculographic analysis of word reading in hemispatial neglect. Physiology \& Behavior. 77, 613-619.

Benton, A.L., Hamsher, K.d., Varney, N.R., Spreen, O., 1983. Contributions to Neuropsychological Assessment. A Clinical Manual. Vol., Oxford University Press, New York.

Binder, J.R., Lazar, R.M., Tatemichi, T.K., Mohr, J.P., Desmond, D.W., Ciecierski, K.A., 1992. Left hemiparalexia. Neurology. 42, 562-569.

Caramazza, A., Hillis, A.E., 1990. Spatial representation of words in the brain implied by studies of a unilateral neglect patient. Nature. 346, 267-269.

Chao, L.L., Haxby, J.V., Martin, A., 1999. Attribute-based neural substrates in temporal cortex for perceiving and knowing about objects. Nature Neuroscience. 2, 913-919.

Chechlacz, M., Rotshtein, P., Bickerton, W.L., Hansen, P.C., Deb, S., Humphreys, G.W., 2010. Separating neural correlates of allocentric and egocentric neglect: Distinct cortical sites and common white matter disconnections. Cognitive Neuropsychology. 27, 277-303.

Costello, A.D., Warrington, E.K., 1987. The dissociation of visuospatial neglect and neglect dyslexia. Journal of Neurology, Neurosurgery, and Psychiatry. 50, 1110-1116.

Cubelli, R., Nichelli, P., Bonito, V., De Tanti, A., Inzaghi, M.G., 1991. Different patterns of dissociation in unilateral spatial neglect. Brain and Cognition. 15, 139-159.

Daducci, A., Gerhard, S., Griffa, A., Lemkaddem, A., Cammoun, L., Gigandet, X., Meuli, R., Hagmann, P., Thiran, J.P., 2012. The connectome mapper: an open-source processing pipeline to map connectomes with MRI. PLoS One. 7, e48121.

Desikan, R.S., Segonne, F., Fischl, B., Quinn, B.T., Dickerson, B.C., Blacker, D., Buckner, R.L., Dale, A.M., Maguire, R.P., Hyman, B.T., Albert, M.S., Killiany, R.J., 2006. An automated labeling system for subdividing the human cerebral cortex on MRI scans into gyral based regions of interest. Neuroimage. 31, 968-80. 
Ellis, A.W., Flude, B.M., Young, A.W., 1987. "Neglect dyslexia" and the early visual processing of letters in words and nonwords. Cognitive Neuropsychology. 4, 439-464.

Fisher, D.F., 1975. Reading and visual search. Mem Cognit. 3, 188-96.

Friedrich, F.J., Egly, R., Rafal, R.D., Beck, D., 1998. Spatial attention deficits in humans: A comparison of superior parietal and temporal-parietal junction lesions. Neuropsychology. 12, 193-207.

Gauthier, L., Dehaut, F., Joanette, Y., 1989. The Bells Test: A quantative and qualitative test for visual neglect. International Journal of Clinical Neuropsychology. 11, 49-54.

Golay, L., Schnider, A., Ptak, R., 2008. Cortical and subcortical anatomy of chronic spatial neglect following vascular damage. Behavioral and Brain Functions. 4, 43.

Grimsen, C., Hildebrandt, H., Fahle, M., 2008. Dissociation of egocentric and allocentric coding of space in visual search after right middle cerebral artery stroke. Neuropsychologia. 46, 902914.

Guggisberg, A.G., Rizk, S., Ptak, R., Di Pietro, M., Saj, A., Lazeyras, F., Lovblad, K.-O., Schnider, A., Pignat, J.-M., 2015. Two intrinstic coupling types for resting-state integration in the human brain. Brain Topogr. 28, 318-329.

Halligan, P.W., Fink, G.R., Marshall, J.C., Vallar, G., 2003. Spatial cognition: evidence from visual neglect. Trends in Cognitive Sciences. 7, 125-133.

Haywood, M., Coltheart, M., 2001. Neglect dyslexia with a stimulus-centred deficit and without visuospatial neglect. Cognitive Neuropsychology. 18, 577-615.

Hillis, A.E., Caramazza, A., 1991. Deficit to stimulus-centered, letter shape representations in a case of "unilateral neglect". Neuropsychologia. 29, 1223-40.

Hillis, A.E., Caramazza, A., 1995. A framework for interpreting distinct patterns of hemispatial neglect. Neurocase. 1, 189-207.

Hillis, A.E., Rapp, B., Benzing, L., Caramazza, A., 1998. Dissociable coordinate frames of unilateral spatial neglect: "viewer-centered" neglect. Brain and Cognition. 37, 491-526.

Hillis, A.E., 2006. Neurobiology of unilateral spatial neglect. Neuroscientist. 12, 153-63.

Holm, S., 1979. A simple sequentially rejective multiple test procedure. Scandinavian Journal of Statistics. 6, 65-70. 
Humphreys, G.W., Riddoch, M.J., 1995. Separate coding of space within and between perceptual objects: evidence from unilateral visual neglect. Cognitive Neuropsychology. 12, 283-311.

Humphreys, G.W., 1998. Neural representation of objects in space: a dual coding account. Philosophical Transactions of the Royal Society of London B. 353, 1341-1351.

Indovina, I., Macaluso, E., 2007. Dissociation of stimulus relevance and saliency factors during shifts of visuospatial attention. Cerebral Cortex. 17, 1701-1711.

Katz, R.B., Sevush, S., 1989. Positional dyslexia. Brain and Language. 37, 266-289.

Kincade, J.M., Abrams, R.A., Astafiev, S.V., Shulman, G.L., Corbetta, M., 2005. An event-related functional magnetic resonance imaging study of voluntary and stimulus-driven orienting of attention. Journal of Neuroscience. 25, 4593-604.

Làdavas, E., Shallice, T., Zanella, M.T., 1997. Preserved semantic access in neglect dyslexia. Neuropsychologia. 35, 257-270.

Lee, B.H., Suh, M.K., Kim, E.J., Seo, S.W., Choi, K.M., Kim, G.M., Chung, C.S., Heilman, K.M., Na, D.L., 2009. Neglect dyslexia: frequency, association with other hemispatial neglects, and lesion localization. Neuropsychologia. 47, 704-10.

Losier, B.J.W., Klein, R.M., 2001. A review of the evidence for a disengage deficit following parietal lobe damage. Neuroscience and Biobehavioral Reviews. 25, 1-13.

Marr, D., 1982. Vision. Vol., Freeman and Co., San Francisco.

Martelli, M., Arduino, L.S., Daini, R., 2011. Two different mechanisms for omission and substitution errors in neglect dyslexia. Neurocase. 17, 122-32.

Medina, J., Kannan, V., Pawlak, M.A., Kleinman, J.T., Newhart, M., Davis, C., Heidler-Gary, J.E., Herskovits, E.H., Hillis, A.E., 2009. Neural substrates of visuospatial processing in distinct reference frames: evidence from unilateral spatial neglect. Journal of Cognitive Neuroscience. 21, 2073-2084.

Miceli, G., Capasso, R., 2001. Word-centred neglect dyslexia: evidence from a new case. Neurocase. 7, 221-237.

Molenberghs, P., Gillebert, C.R., Peeters, R., Vandenberghe, R., 2008. Convergence between lesion-symptom mapping and functional magnetic resonance imaging of spatially selective attention in the intact brain. Journal of Neuroscience. 28, 3359-73. 
Morrow, L.A., Ratcliff, G., 1988. The disengagement of covert attention and the neglect syndrome. Psychobiology. 16, 261-269.

New, B., Pallier, C., Brysbaert, M., Ferrand, L., 2004. Lexique 2: A new French lexical database. Behavior Research Methods, Instruments \& Computers. 36, 516-524.

Oldfield, R.C., 1971. The assessment and analysis of handedness: the Edinburgh inventory. Neuropsychologia. 9, 97-113.

Patterson, K., Wilson, B., 1990. A rose is a rose or a nose: A deficit in initial letter identification. Cognitive Neuropsychology. 7, 447-477.

Primativo, S., Arduino, L.S., De Luca, M., Daini, R., Martelli, M., 2013. Neglect dyslexia: a matter of "good looking". Neuropsychologia. 51, 2109-19.

Primativo, S., Arduino, L.S., Daini, R., De Luca, M., Toneatto, C., Martelli, M., 2015. Impaired oculomotor behaviour affects both reading and scene perception in neglect patients. Neuropsychologia. 70, 90-106.

Ptak, R., Schnider, A., Golay, L., Müri, R., 2007. A non-spatial bias favouring fixated stimuli revealed in patients with spatial neglect. Brain. 130, 3211-3222.

Ptak, R., Di Pietro, M., Schnider, A., 2012. The neural correlates of object-centered processing in reading: A lesion study of neglect dyslexia. Neuropsychologia. 50, 1142-1150.

Ptak, R., Fellrath, J., 2013. Spatial neglect and the neural coding of attentional priority. Neuroscience and Biobehavioral Reviews. 37, 705-722.

Reinhart, S., Wagner, P., Schulz, A., Keller, I., Kerkhoff, G., 2013. Line bisection error predicts the presence and severity of neglect dyslexia in paragraph reading. Neuropsychologia. 51, 1-7.

Rey, A., 1964. L'Examen Clinique en Psychologie. Vol., Presse Universitaire de France, Paris.

Riddoch, J., Humphreys, G., Cleton, P., Fery, P., 1990. Interaction of attentional and lexical processes in neglect dyslexia. Cognitive Neuropsychology. 7, 479-517.

Ronchi, R., Algeri, L., Chiapella, L., Spada, S., Vallar, G., 2012. Spatial neglect and perseveration in visuomotor exploration. Neuropsychology. 26, 588-603.

Rorden, C., Karnath, H.-O., Bonilha, L., 2007. Improving lesion-symptom mapping. Journal of Cognitive Neuroscience. 19, 1081-1088. 
Rorden, C., Bonilha, L., Fridriksson, J., Bender, B., Karnath, H.O., 2012. Age-specific CT and MRI templates for spatial normalization. Neuroimage. 61, 957-65.

Serences, J.T., Shomstein, S., Leber, A.B., Golay, X., Egeth, H.E., Yantis, S., 2005. Coordination of voluntary and stimulus-driven attentional control in human cortex. Psychol Sci. 16, 114-22.

Shulman, G.L., Astafiev, S.V., Franke, D., Pope, D.L., Snyder, A.Z., McAvoy, M.P., Corbetta, M., 2009. Interaction of stimulus-driven reorienting and expectation in ventral and dorsal frontoparietal and basal ganglia-cortical networks. Journal of Neuroscience. 29, 4392-407.

Stenneken, P., van Eimeren, L., Keller, I., Jacobs, A.M., Kerkhoff, G., 2008. Task-dependent modulation of neglect dyslexia? Novel evidence from the viewing position effect. Brain Res. 1189, 166-78.

Subbiah, I., Caramazza, A., 2000. Stimulus-centered neglect in reading and object recognition. Neurocase. 6, 13-31.

Ungerleider, L.G., Mishkin, M., 1982. Two cortical visual systems. In Analysis of visual behavior. Vol., D.J. Ingle, M.A. Goodale, R.J.W. Mansfield, ed.^eds. MIT Press, Cambridge, pp. 549-586.

Ungerleider, L.G., Pasternak, T., 2003. Ventral and dorsal cortical processing streams. In The Visual Neurosciences. Vol., L.M. Chalupa, J.S. Werner, ed.^eds. MIT Press, Boston, pp. 541-562.

Vallar, G., Burani, C., Arduino, L.S., 2010. Neglect dyslexia: a review of the neuropsychological literature. Experimental Brain Research. 206, 210-235.

Warrington, E., 1984. Recognition memory test. Vol., NFER-Nelson, Windsor, UK.

Warrington, E.K., James, M., 1991. The Visual Object and Space Perception Battery. Vol., Thames Valley Test Company, Bury St Edmunds.

Wechsler, D., 2008. The Wechsler Adult Intelligence Scale - Fourth Edition. Vol., Pearson, San Antonio, TX.

Zimmermann, P., Fimm, B., 2010. TAP. Tests d'Evaluation de l'Attention. Vol., Psytest, Herzogenrath, Germany. 
Table 1. Neuropsychological scores in visual-perceptual tests.

\begin{tabular}{|c|c|c|}
\hline Test & Score & Percentile \\
\hline \multicolumn{3}{|c|}{ Visual object and space perception battery $^{1}$} \\
\hline Incomplete letters & 16 & 1 \\
\hline Silhouettes & 17 & 7 \\
\hline Object decision & 18 & 35 \\
\hline Progressive silhouettes & 11 & 31 \\
\hline Dot counting & 8 & 1 \\
\hline Position discrimination & 16 & 1 \\
\hline Number location & 8 & 10 \\
\hline Cube analysis & 7 & 3 \\
\hline Judgment of line orientation ${ }^{2}$ & 25 & 56 \\
\hline Bells test, left omissions ${ }^{3}$ & 1 & - \\
\hline Bells test, right omissions & 2 & - \\
\hline Letter cancellation, left omissions ${ }^{4}$ & 0 & - \\
\hline Letter cancellation, right omissions & 0 & - \\
\hline Line bisection, mean error in $\mathrm{mm}^{5}$ & 5 & 1 \\
\hline Posner, left-valid (ms) & 374 & 12 \\
\hline Posner, left-invalid (ms) & 321 & 66 \\
\hline Posner, right-valid (ms) & 382 & 10 \\
\hline Posner, right-invalid (ms) & 405 & 12 \\
\hline Rey complex figure, copy ${ }^{6}$ & 32 & 45 \\
\hline WAIS, Block design ${ }^{7}$ & 24 & 5 \\
\hline
\end{tabular}




\section{Figure legends}

Figure 1. Humphrey perimetry of DM's visual fields (only results for the right eye are shown). The left shows the central \pm 30 degrees. On the right results of more detailed testing of the central \pm 10 degrees are shown. The row of X's shows the size of projection of a six-letter word.

Figure 2. Results of DM's reading. Rates of different types of neglect dyslexia errors as a function of lexical status of the stimulus (A). Within-word position of reading errors for 6-letter words, as a function of the location of the word on screen (B; central vs. 6 degrees left or right of fixation). Withinword positions were defined as follows: left - first two letters of the word; centre - central two letters; right - last two letters.

Figure 3. Structural imaging findings. Left, posterior and right view of DM's brain (A). Sagittal slices through the left $(B)$ and the right hemisphere $(C)$.

Figure 4. Visualization of the number of fibres in the brain of a representative control subject and patient DM. Left hemisphere connections between the supramarginal and superior temporal gyrus (A); the supramarginal and middle temporal gyrus (B); the inferior parietal region and the superior temporal gyrus (C); and the inferior parietal region and the middle temporal gyrus (D). Right hemisphere connections between the supramarginal and the middle temporal gyrus $(E)$ and the inferior parietal region and the middle temporal gyrus $(F)$. 


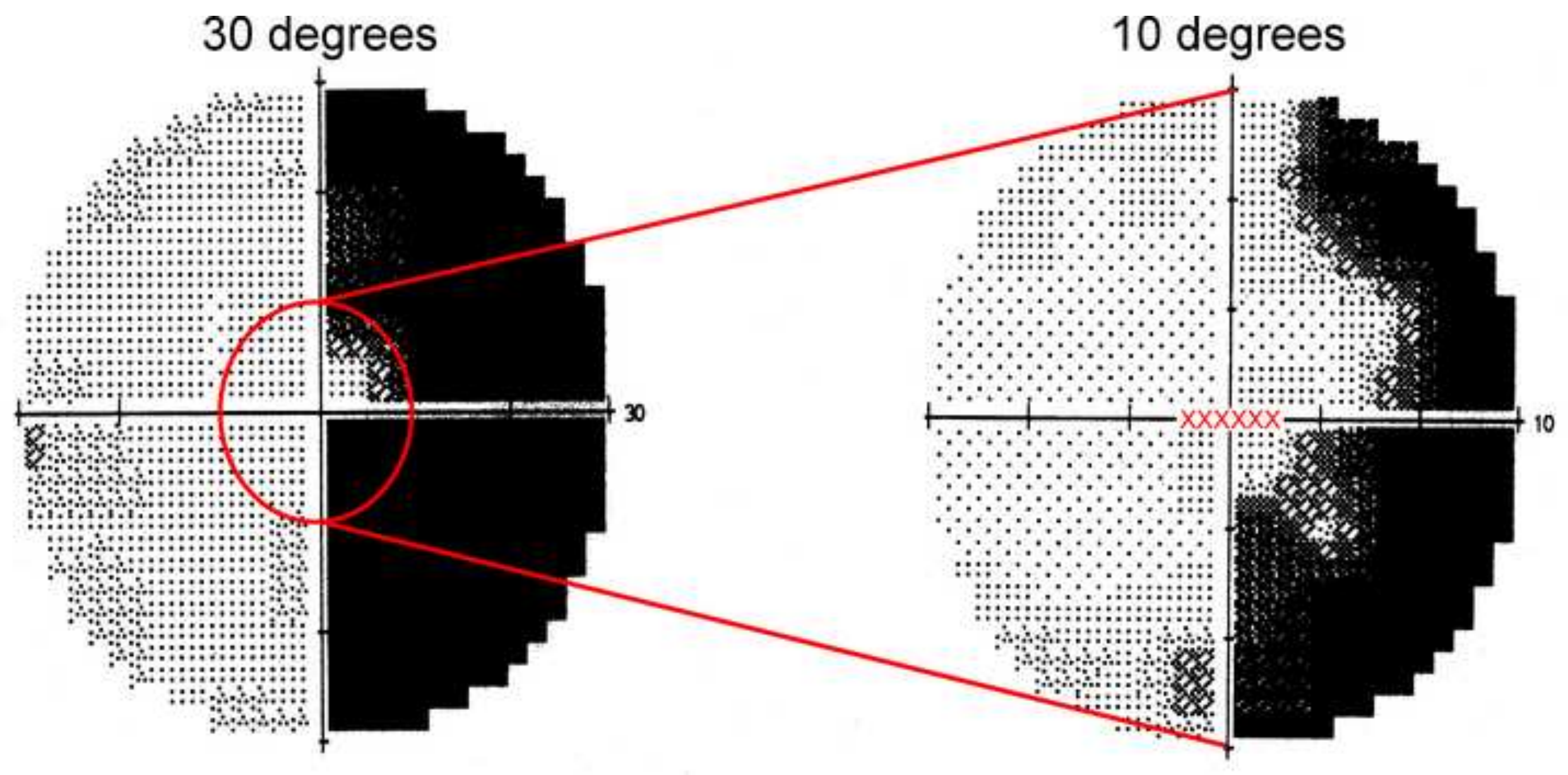


A)

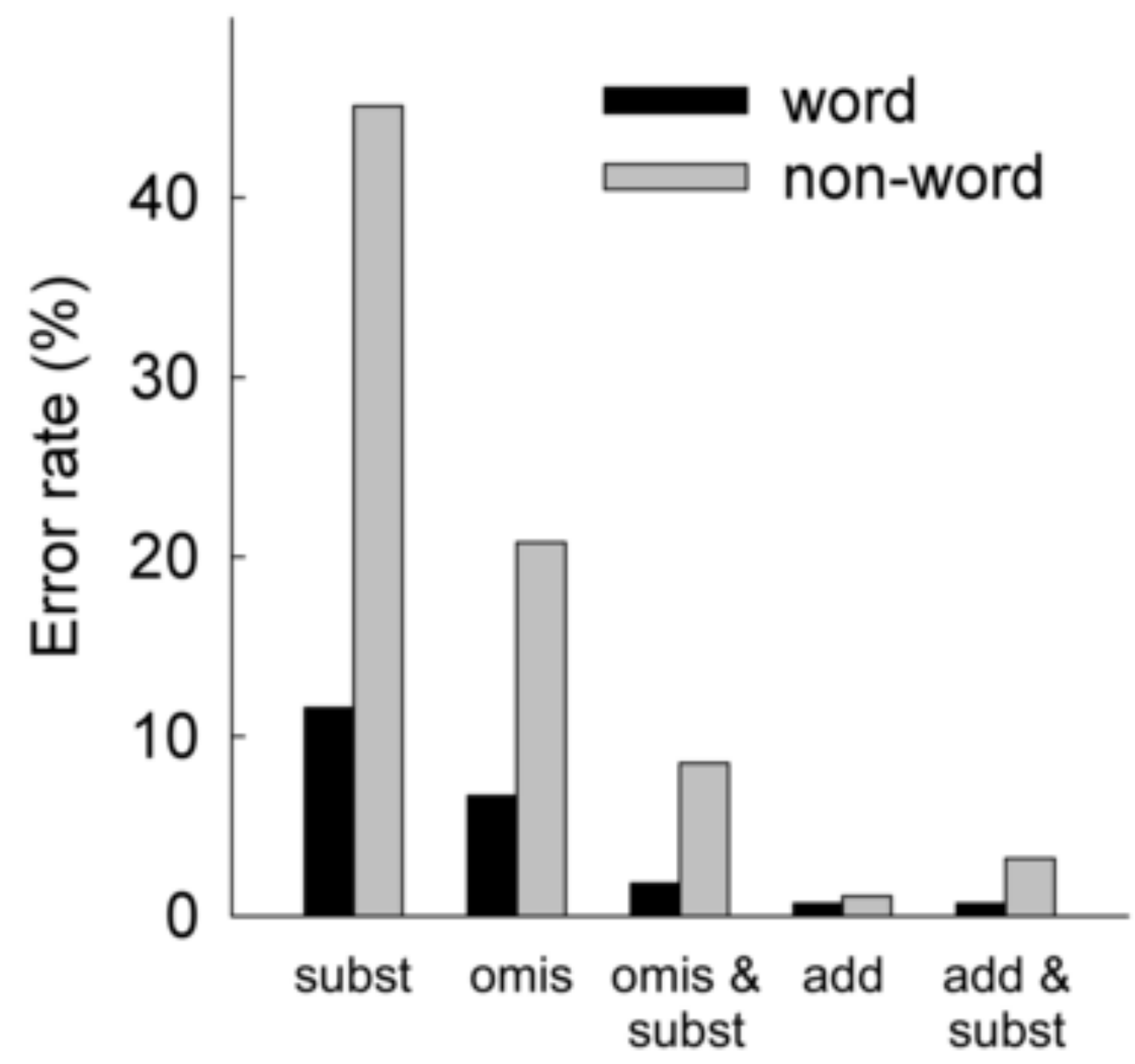

B)

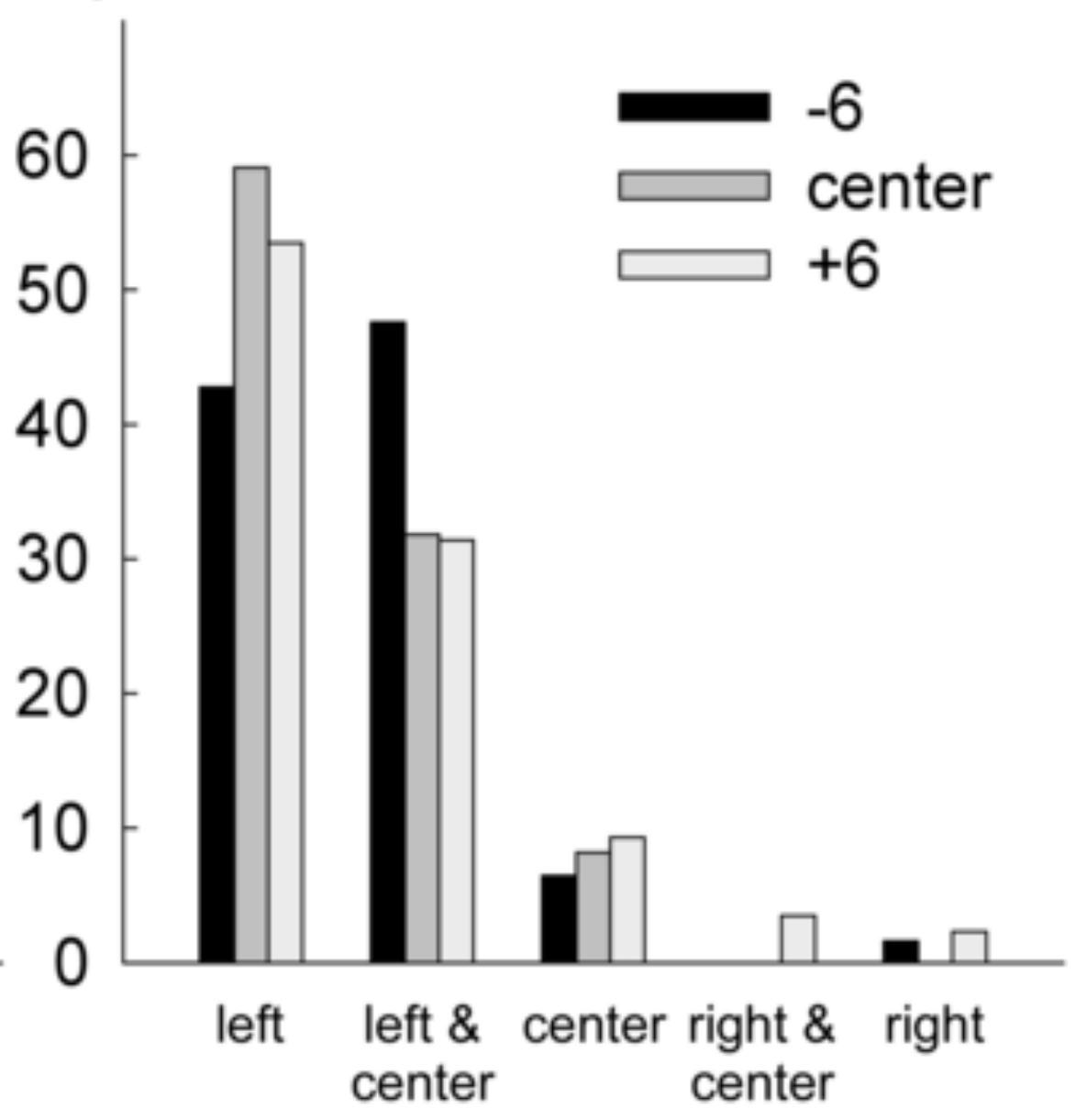


A)
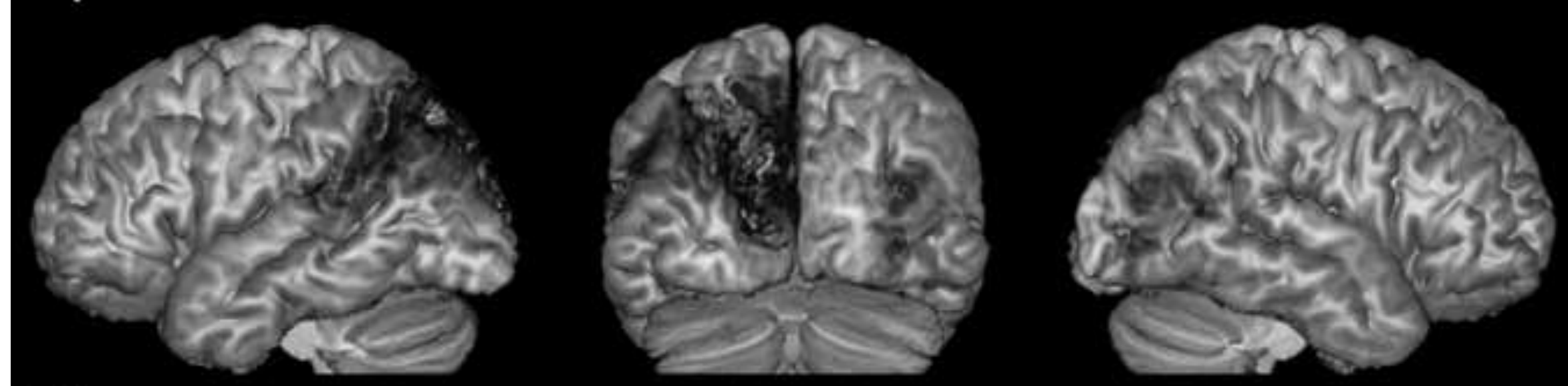

B)

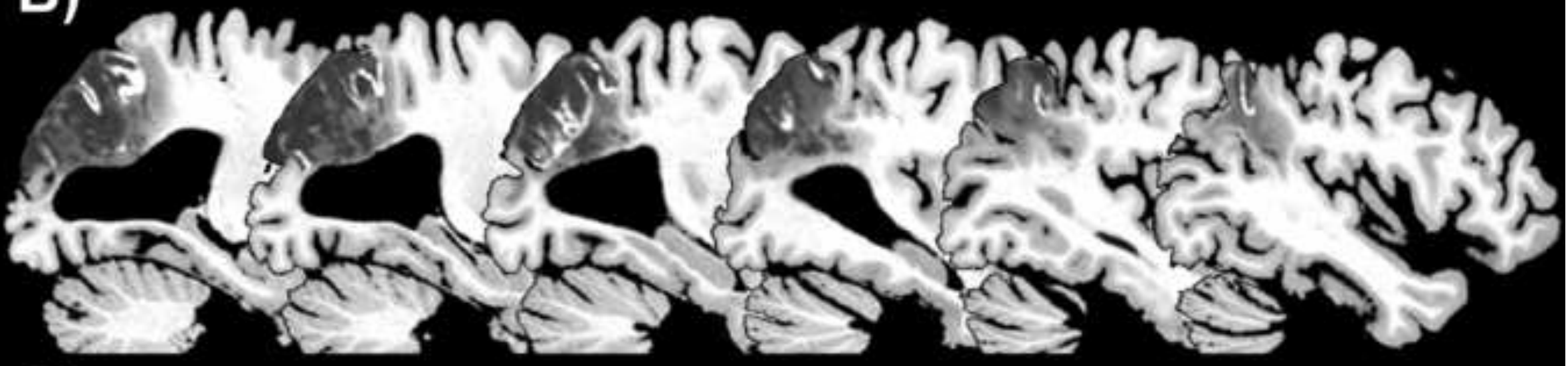

C)

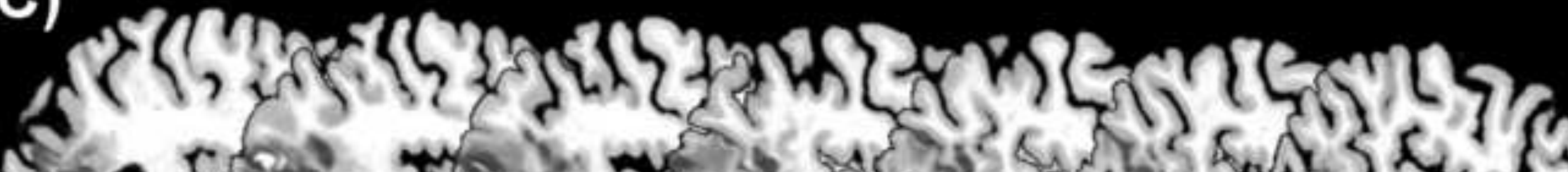

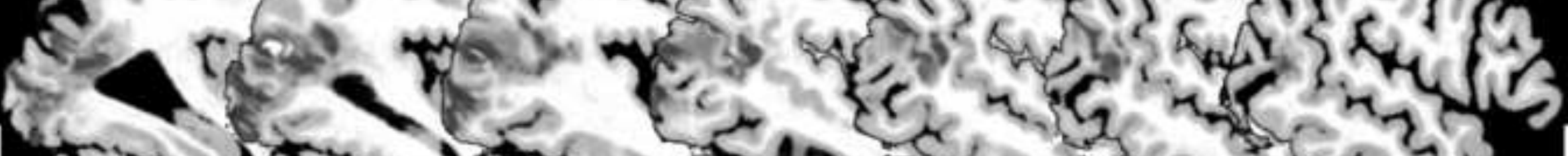
$5 .+5$. 

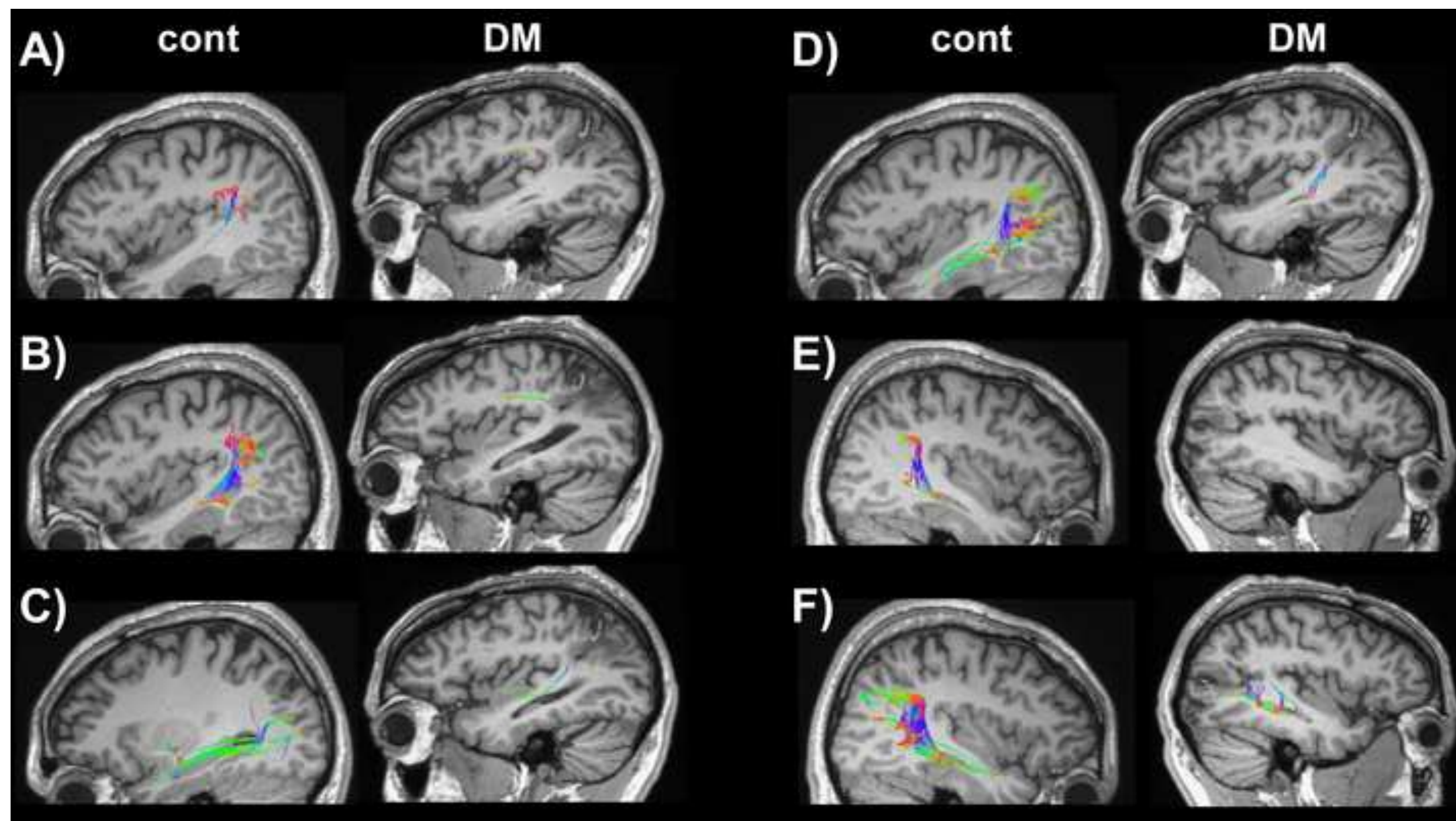

F)
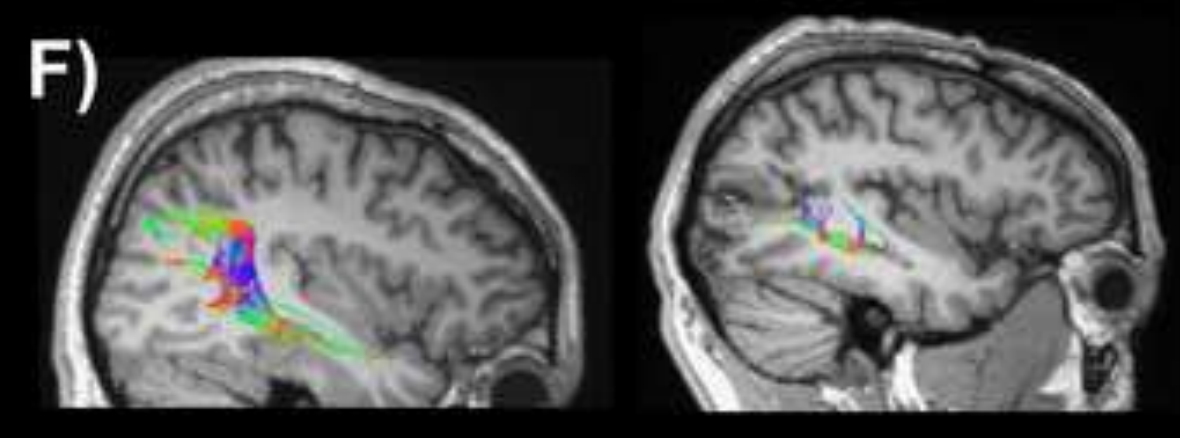